model. In the Fröhlich-Kennelly model, magnetic permeability smoothes out over the whole particle. Its peak value drops below $20 \%$ of its original's even at low applied fields $\left(\mu_{0} * H_{0}=0.05\right.$ Tesla). For the Piecewise Linear model, the permeability of the core of the particle remains $\mu_{F e}$ as the region between the core and fluid becomes saturated. This is the essential difference of the two models.

Finally, we compute the intrinsic inductions for $\mathrm{MR} \mathrm{flu-}$ ids using the these models and then compare them with the experimental results conducted at the Lord Corporation. The intrinsic induction represents the overall magnetization for MR fluids and its formulations are described in [2]-[5]. In figure 4, we present the magnetic intrinsic induction for three different samples of $10 \%, 20 \%$ and $30 \%$ particle area fractions. These results are in remarkable agreement.
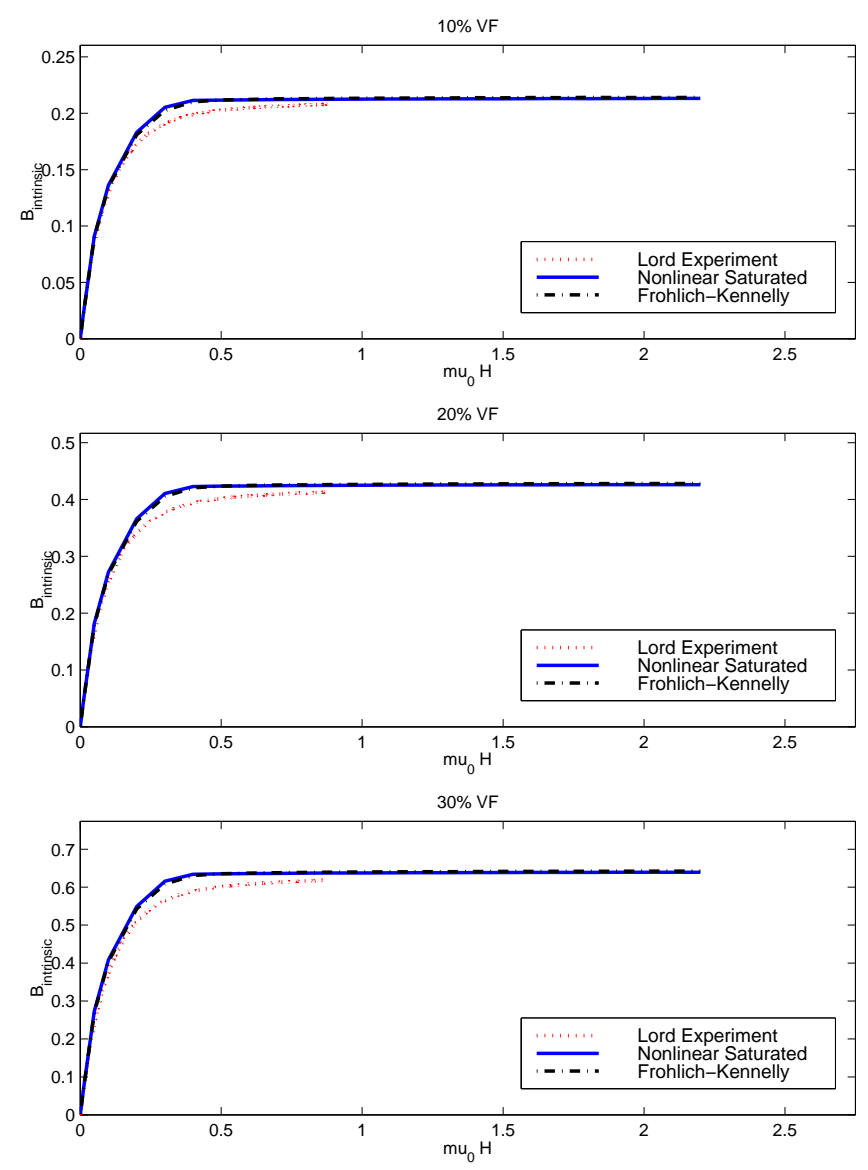

Fig. 4. Magnetic Intrinsic Inductions of the Piecewise Linear Model and the Fröhlich-Kennelly Model Are Compared to Experimental Results Conducted at the Lord Corporation

Remark: Deducing the nonlinear saturation law by comparing macroscopic magnetic induction/applied magnetic field curves as we have done here is equivalent to solving an inverse problem. In this case, the observations are the values of magnetic induction, the model is the nonlinear constitutive law between magnetic induction and applied magnetic field, and the unknown is the form of the nonlinearity related to magnetic saturation in the model. Viewing our comments in this manner and recalling the well-known ill-posedness (e.g. lack of unique solution) of many inverse problems (see [6], [7] for examples and general discussions of this and more general aspects of the ill-posed nature of the inverse problems), it is not surprising that we are able to achieve relatively good fits to the observed data using two very different saturation laws (see equations (4), (6) and Figure 1). In general, one cannot expect a unique constitutive law from such procedures unless one adds further physical constraints (based, for example, on hypothesized mechanicms such as a magnetic domain wall theory) to restrict the class of nonlinearities in the estimation or inverse problem.

\section{Summary and Conclusions}

We have examined two magnetic saturation models for the ferrous particle phase of magnetorheological fluids using Finite Element Analysis. One model is the well-known Fröhlich-Kennelly model and the other is the Piecewise Linear model. The discrepancy between the smooth and piecewise models is evident when examining the microscopic scale. However, the overall magnetic properties of magnetorheological composite are relatively insensitive to the form of the magnetic saturation model for the particular phase. This may suggest that the overall magnetic properties of such composites are largely dictated by the growth of the saturated areas from the polar region of the particles.

\section{ACKNOWLEDGMENTS}

This work was supported jointly by the National Science Foundation Industrial Post-Doctoral Grant (No. DMS9704963) and by the Lord Corporation through funds to the Center for Research in Scientific Computation at North Carolina State University. Part of this research was done when C. H. Lee (Hung V. Ly) was a National Science Foundation Industrial Post-Doctoral Fellow at the Center for Research in Scientific Computation- North Carolina State University. F. Reitich gratefully acknowledges support from AFOSR through grant No. F49620-95-1-0113 and from NSF through grant No. DMS-9622555. H.T. Banks was supported in part by AFOSR under grants F49620-951-0236 and F49620-98-1-0180.

\section{REFERENCES}

[1] R. M. Bozorth, Ferromagnetism, van Nostrand, Toronto (1951). [2] T. Simon, F. Reitich, M. R. Jolly, K. Ito, and H. T. Banks, "Estimation of the Effective Permeability in Magnetorheological Fluids," CRSC TR-98-35, North Carolina State University, (1998); Journal of Intelligent Material Systems and Structures (submitted).

[3] H. V. Ly, F. Reitich, M. Jolly, H. T. Banks, and K. Ito, "Simulations of Particle Dynamics for Magnetorheological Fluids," Jounal of Computational Physics, 155 (1999) pp. 160-177.

[4] H. V. Ly, F. Reitich, M. Jolly, H. T. Banks, and K. Ito, "Parameter Studies for the Magnetorheological Fluids Using the Fast Multipole Method," in preparation, (1999).

[5] H. T. Banks, K. Ito, M. Jolly, H. V. Ly, F. Reitich, and T. M. Simon, "Dynamic Simulations and Nonlinear Homogenization Study for Magnetorheological Fluids," Smart Structures and Materials: Mathematics and Control in Smart Materials, (1999) pp. $92-100$.

[6] H. T. Banks and K. Kunisch, Estimation Techniques for Distributed Parameter Systems, Birkhaüser, Boston, (1989).

[7] H. T. Banks, R. C. Smith and Y. Wang, Smart Material Structures: Modeling, Estimation, and Control, Masson and John Wiley, Paris/Chichester, (1996). 

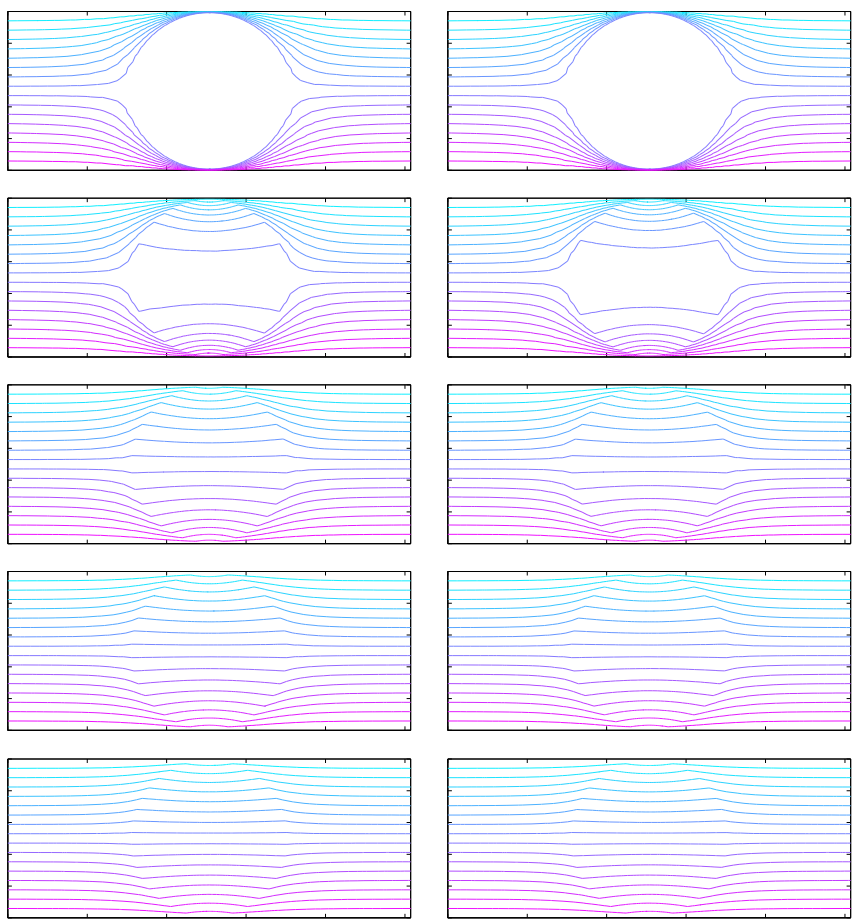

Fig. 2. Equi-potential curves for the Fröhlich-Kennelly model (left) and the Piecewise Linear model (right) with applied fields 0.01 Tesla, 0.4 Tesla, 0.9 Tesla, 1.4 Tesla, 2.2 Tesla from top to bottom, respectively.

\section{Numerical Results and Comparisons}

For the composites we consider, no free currents are present in the domain. Thus the second equation in (1) becomes $\nabla \times \overrightarrow{\mathbf{H}}=0$ which, in turn, implies that the magnetic field can be written in terms of a scalar potential $\phi$,

$$
\overrightarrow{\mathbf{H}}=-\nabla \phi .
$$

As a result, equations (1)-(3) can be simplified to

$$
\nabla \cdot(\mu(|\nabla \phi|) \nabla \phi)=0
$$

with $\mu(|\nabla \phi|)$ given by (3) and (4) or (6). We assume that the external applied field is in the vertical direction $\overrightarrow{\mathbf{H}}_{0}=H_{0} \overrightarrow{\mathbf{e}}_{2}$ with intensity $H_{0}$. The particles are assumed to be circular and arranged periodically in both horizontal and vertical directions. As a result, the magnetic field is periodic and it is natural to assume the MR fluid sample consists of one particle. In our numerical studies, the dimensions of the cell are chosen so that the circular particle constitutes certain area fraction of the rectangular cell and the inter-particle distance is always kept at $1.6 \%$ of the particle radius. We let $L_{x} \times L_{y}$ be the dimensions of the cell in $\mathbb{R}^{2}$ and we require the scalar potential $\phi$ to satisfy the following Dirichlet boundary conditions

$$
\phi(x, y)=\left\{\begin{array}{cc}
H_{0} L_{y} & 0 \leq x \leq L_{x}, y=0, \\
H_{0} L_{y}\left(1-\frac{y}{L_{y}}\right) & 0 \leq y \leq L_{y}, x=L_{x}, \\
0 & 0 \leq x \leq L_{x}, y=L_{y}, \\
H_{0} L_{y}\left(1-\frac{y}{L_{y}}\right) & 0 \leq y \leq L_{y}, x=0 .
\end{array}\right.
$$

Mathematical formulations for magnetic fields and scalar potentials including effective permeability and homogenization can be found in [3], [4], [5].

To obtain the scalar potential $\phi$, we solve equations (8)-(9) using the finite element method. The fluid permeability is assumed to be the free-space permeability $\left(\mu_{0}=4 \pi \times 10^{-7} N / A^{2}\right)$ and $\mu_{F e}=2000 \times \mu_{0}$. In the Fröhlich-Kennelly model, $B_{s}=2.15$ Tesla is used in equation (4) and $H_{c}=0.13375 \mathrm{~A} / \mathrm{m}$ is used in equation (6) for the Piecewise Linear model. The resulting equi-potential curves for the Fröhlich-Kennelly model and the Piecewise Linear model are plotted in Figure 2.

\section{Frohlich-Kennelly Model}

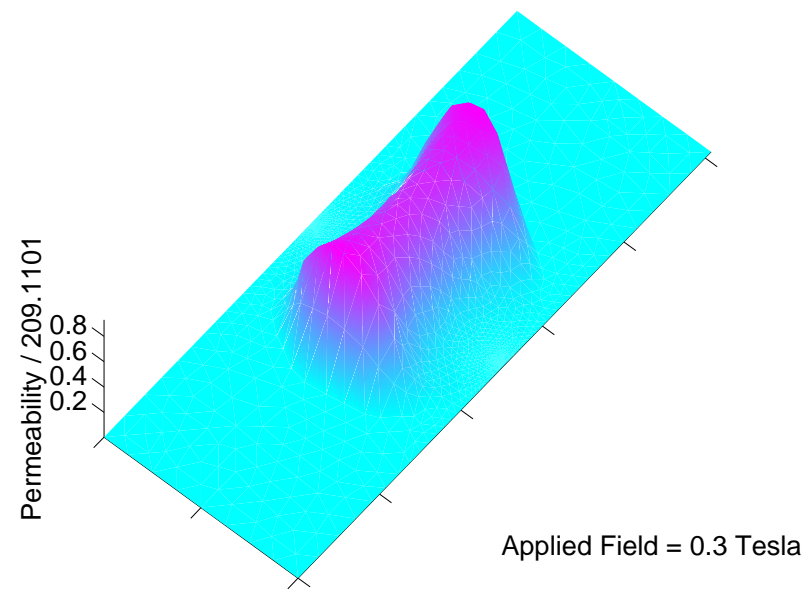

Piecewise Linear Model

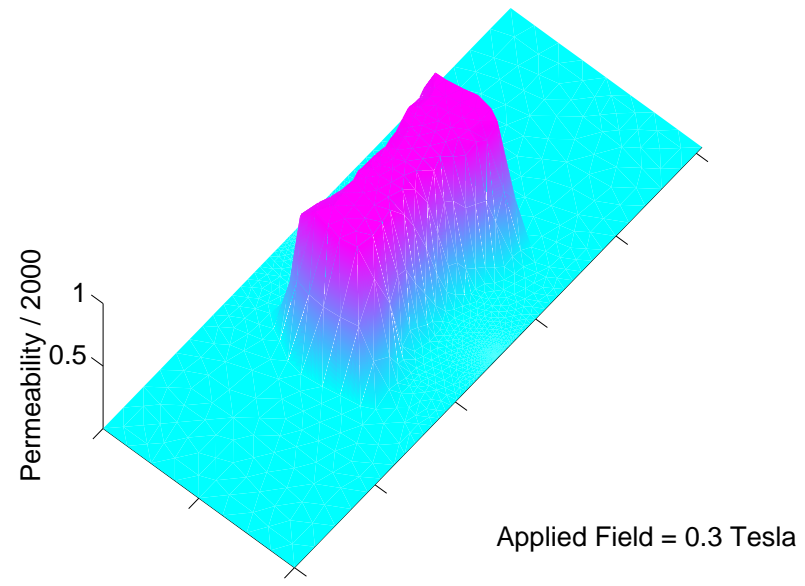

Fig. 3. Top: Fröhlich-Kennelly mode; the permeability smoothes out over the domain of the particle as it saturates; almost $90 \%$ of the value of the permeability has been lost. Bottom: Piecewise Linear model; the core of the particle remains iron while the region between the core and fluid becomes saturated.

Even though the equi-potential curves are identical, the magnetic saturation of the two models are substantially different. Figure 3 depicts a profile of the permeability for the Fröhlich-Kennelly model and the Piecewise Linear 


\title{
A Piecewise Linear Model for Field-Responsive Fluids
}

\author{
Charles H. Lee, Fernando Reitich, Mark R. Jolly, H. Thomas Banks and Kazifumo Ito
}

\begin{abstract}
The Fröhlich-Kennelly model provides a constitutive law which is field-dependent and has been widely used for studying nonlinear properties for a variety of electric and magnetic applications. Under the Fröhlich-Kennelly model, saturation begins to occupy the entire conducting domain even at low-moderate applied fields. In this paper, we first present a new nonlinear constitutive law for field-responsive fluids which depends on the local fields and allows regions where the fields have not reached a critical value to remain unsaturated. We then study numerically the nonlinear saturated model and compare the results to the Fröhlich-Kennelly model and experiments performed at the Lord Corporation.
\end{abstract}

Keywords - Field-Responsive Fluids, Nonlinear Saturation, Fröhlich-Kennelly and Piecewise Linear Models.

\section{Constituent Laws}

In this section and the remainder of the paper, we will address all terminology in terms of magnetostatics for magnetically-dependent magnetorheological fluids. The same context can be written in terms of electrostatics in discussion for electrorheological fluids. Let us consider a container $\Omega$ which is filled with a non-magnetic viscous fluid and $M$ ferrous particles $\Omega_{1}, \Omega_{2}, \cdots, \Omega_{M}$, where $M$ is very large. Then the field is governed by the equations of magnetostatics, namely,

$$
\nabla \cdot \overrightarrow{\mathbf{B}}=0, \quad \text { and } \quad \nabla \times \overrightarrow{\mathbf{H}}=\overrightarrow{\mathbf{J}},
$$

where $\overrightarrow{\mathbf{J}}$ is the free current, $\overrightarrow{\mathbf{H}}$ is the magnetic field and $\overrightarrow{\mathbf{B}}$ is the magnetic induction. The material constitutive relationship that we consider is of the following form,

$$
\overrightarrow{\mathbf{B}}=\overrightarrow{\mathbf{B}}_{i}+\mu_{0} \overrightarrow{\mathbf{H}}=\mu \overrightarrow{\mathbf{H}},
$$

where $\overrightarrow{\mathbf{B}}_{i}$ is the intrinsic induction caused by magnetization, $\mu_{0}$ is the permeability of free space and

$$
\mu= \begin{cases}\mu_{k} & \text { in the kth particle, } \\ \mu_{0} & \text { in the carrier oil. }\end{cases}
$$

For low external applied fields, $\mu_{k}$ may be approximated as a constant. However, in general, $\mu_{k}$ is a function of $\overrightarrow{\mathbf{H}}$ in order to model magnetic saturation. In the next two subsections, we will describe two nonlinear constitutive laws for saturation: the Fröhlich-Kennelly[1] model and a piecewise linear model which we propose as an alternative.

Charles Hung Lee (Hung V. Ly) was with the Center for Research in Scientific Computation, North Carolina State University, Raleigh, NC 27695-8205. He is now with the Department of Mathematics, California State University, Fullerton, CA 92834, USA.

Fernando Reitich is with the School of Mathematics, University of Minnesota, Minneapolis, MN 55455.

Mark R. Jolly is with the Material Division, Lord Corporation, 110 Lord Drive, Cary, NC 27511.

H. Thomas Banks and Kazifumo Ito are with the Center for Research in Scientific Computation, North Carolina State University, Raleigh, NC 27695-8205.

\section{A. Fröhlich-Kennelly Model}

The Fröhlich-Kennelly model was described in [1] and is given by

$$
\mu_{k}(|\overrightarrow{\mathbf{H}}|)=\mu_{0}+\frac{\left(\mu_{F e}-\mu_{0}\right) B_{s}}{\left(\mu_{F e}-\mu_{0}\right)|\overrightarrow{\mathbf{H}}|+B_{s}}
$$

where $B_{s}$ denotes the magnetic saturation and $\mu_{F e}$ denotes the permeability of iron. Numerical studies for MR fluids using this model can be found in [2].

\section{B. Piecewise Linear Model}

In the permeable region, the material constituents that we propose satisfy the following relation

$$
\overrightarrow{\mathbf{B}}= \begin{cases}\mu_{F e} \overrightarrow{\mathbf{H}} & |\overrightarrow{\mathbf{H}}|<H_{c} \\ \mu_{0} \overrightarrow{\mathbf{H}}+\left(\mu_{F e}-\mu_{0}\right) H_{c} \frac{\overrightarrow{\mathbf{H}}}{|\overrightarrow{\mathbf{H}}|} & |\overrightarrow{\mathbf{H}}| \geq H_{c}\end{cases}
$$

where the critical value $H_{c}$ is a fixed scalar constant. As a result, the permeability in the kth particle, $\mu_{k}$ in equation (3), can be expressed locally as

$$
\mu_{k}(|\overrightarrow{\mathbf{H}}|)= \begin{cases}\mu_{F e} & |\overrightarrow{\mathbf{H}}|<H_{c}, \\ \mu_{0}+\left(\mu_{F e}-\mu_{0}\right) \frac{H_{c}}{|\overrightarrow{\mathbf{H}}|} & |\overrightarrow{\mathbf{H}}| \geq H_{c} .\end{cases}
$$

Note that in the permeable domain, i.e. the particle, the Piecewise Linear model (6) treats any region whose local fields are below $H_{c}$ as iron.

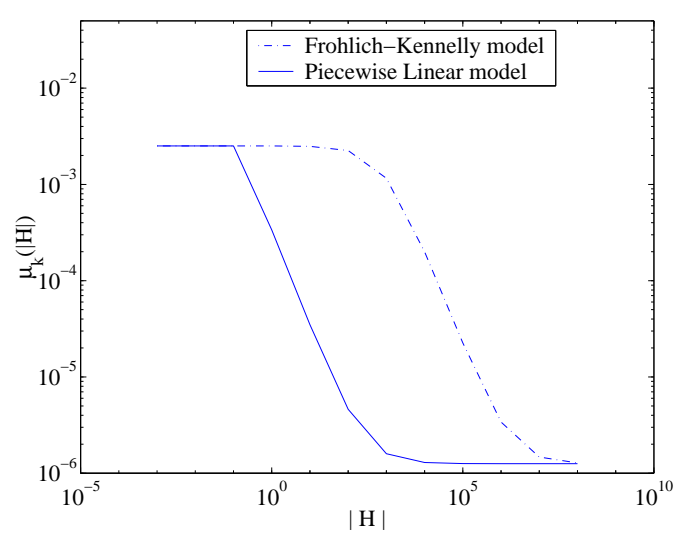

Fig. 1. Particle permeability of the Fröhlich-Kennelly model versus the Piece-wise Linear model 